\title{
Domiciliary non-invasive ventilation in stable COPD?
}

\author{
Mark W Elliott
}

Chronic obstructive pulmonary disease (COPD) is the commonest indication for domiciliary non-invasive ventilation (NIV) in Europe. ${ }^{1}$ However, there is a paucity of evidence to support its use. A number of short-term randomised controlled trials (RCTs) failed to show any consistent benefit from NIV. ${ }^{2-5}$ In a crossover study comparing 3 months of bilevel ventilation plus long term oxygen therapy (LTOT) with LTOT alone, ${ }^{6}$ there were small improvements in arterial blood gas tensions during spontaneous breathing by day and in health-related quality of life (QOL) with NIV. In a longer term RCT, 20 patients randomised to NIV were compared with 24 controls; there was a reduction in the need for hospitalisation in the first 3 months in the NIV group but this effect disappeared by 1 year. One-year survival (78\%) was similar in both groups. Dyspnoea, measured using the Borg scale, was reduced in the NIV group. In a larger RCT from Italy, ${ }^{8} 90$ stable patients on oxygen for more than 6 months were randomly assigned to continuing oxygen alone or oxygen and bilevel ventilation. There was no change in survival, lung or inspiratory muscle function, exercise tolerance or sleep quality score in either group. By contrast, the arterial carbon dioxide tension $\left(\mathrm{PaCO}_{2}\right)$ measured on usual oxygen and resting dyspnoea scores improved. Health-related QOL, as assessed by the Maugeri Foundation Respiratory Failure Questionnaire (MRF 28), improved in the NIV plus oxygen group, but there was no difference in St George's Respiratory Questionnaire (SGRQ) scores. NIV has also been used as an adjunct to pulmonary rehabilitation with two RCTs showing greater improvements in exercise capacity and QOL when NIV, used overnight, was added to a pulmonary rehabilitation programme. ${ }^{9} 10$

In this issue of Thorax McEvoy et al ${ }^{11}$ report the results of an RCT of NIV in patients with chronic stable hypercapnic ventilatory failure due to COPD (see page

Correspondence to: Dr M W Elliott, Department of Respiratory Medicine, St James's University Hospital, Beckett Street, Leeds LS9 7TF, UK; mwelliott@ doctors.org.uk
561). The study was powered for survival. The patients had severe airflow limitation with a forced expiratory volume in $1 \mathrm{~s}\left(\mathrm{FEV}_{1}\right)$ approximately $25 \%$ predicted and severely impaired QOL. The sleep-related increase in transcutaneous carbon dioxide tension $\left(\mathrm{TcCO}_{2}\right)$ was reduced in the NIV + LTOT group compared with the group receiving LTOT alone (12.6 vs $18.8 \mathrm{~mm} \mathrm{Hg}$ ). Over the course of the study there were 40 deaths in the NIV group compared with 46 in the LTOT group. At 24 months, $47 \%$ of the LTOT-treated patients had died compared with $32 \%$ of the NIVtreated patients. By 3.5 years the survival curves had come together. At follow-up at 1 year there was no change in $\mathrm{PaCO}_{2}$ during spontaneous breathing by day on oxygen at the same flow rate in either group or in the $\mathrm{FEV}_{1}$. QOL as measured by the SGRQ was unchanged, but statistically significant differences were observed in several subscales of the SF36 and the profile of mood questionnaires, suggesting that patients treated with NIV had poorer general and mental health, and reported less vigour and more confusion and bewilderment.

It is interesting to compare these data with those previously published (table 1). The rationale behind the use of NIV and the way that it was applied in these studies is different. An understanding of how NIV "works" is critical in determining the therapeutic end point of NIV, but this remains unresolved. ${ }^{12-14}$ Competing theories include resting of chronically fatigued respiratory muscles, reducing the load against which the respiratory muscle pump must work or restoring the central drive to breathe. There is evidence that restoration of central chemosensitivity $^{12}$ and a reduction in load ${ }^{15}$ are important, and the ventilator needs to be set to have an effect upon these parameters. In negative studies, if important physiological parameters are not affected, it cannot be concluded that NIV really had no effect. A major problem with previous studies is that there has either been no clear physiological target behind the choice of ventilator mode and settings or the evidence that an effect has been achieved is weak. Casanova et $\mathrm{al}^{7}$ adjusted pressures to decrease accessory muscle use, reduce the sensation of dyspnoea and reduce respiratory rate by $20 \%$. Oxygen was titrated to maintain saturations of $>90 \%$. No measurements were made of carbon dioxide tensions or data provided confirming the degree to which accessory muscle use or respiratory rate were reduced. No evidence therefore is provided to confirm a physiological effect. Clini et a l $^{8}$ used pressure support ventilation delivered by a spontaneous/timed bilevel ventilator through a nasal mask. Oxygen was added to achieve a target saturation of $>90 \%$. All patients underwent a 10-night NIV trial in hospital before randomisation. Patients were deemed to be compliant when they used the ventilator for $5 \mathrm{~h} /$ night and, among the compliant patients, the average was high at $9.2 \mathrm{~h} /$ night. No data were provided as to the proportion of "compliant" patients or the average compliance overall. The effectiveness of NIV had to be proven by a $5 \%$ decrease in $\mathrm{PaCO}_{2}$ after $1 \mathrm{~h}$ of continuous support and by night time oximetry. NIV was considered effective when the patient spent $>90 \%$ of the recording time with saturations $>90 \%$ during NIV. However, oximetry cannot be used to assess the adequacy of ventilation when patients are receiving supplemental oxygen. ${ }^{16}$ Again, the evidence of a physiological effect from NIV when it was administered (ie, during sleep) is not convincing.

In the study by McEvoy et al, ${ }^{11}$ patients assigned to NIV were admitted for 3-4 days to hospital for education and familiarisation with a patient-triggered bilevel positive pressure device. Bilevel positive airway pressure was gradually increased during daytime and night time trials to the maximum tolerated with a target difference between inspiratory positive airway pressure (IPAP) and expiratory positive airway pressure (EPAP) of $\geqslant 10 \mathrm{~cm} \mathrm{H} H_{2} \mathrm{O}$. The level of EPAP was determined during a polysomnographic study, with the level being increased to abolish snoring and obstructive events. NIV was considered successfully established when at least $3 \mathrm{~h}$ of sleep was confirmed on NIV at an IPAP-EPAP difference of at least $5 \mathrm{~cm} \mathrm{H}_{2} \mathrm{O}$. There was no requirement to change oxygen saturation or $\mathrm{TcCO}_{2}$, although NIV did reduce the maximum sleep-related rise in $\mathrm{TcCO}_{2}$ from 16.5 to $12.6 \mathrm{~mm} \mathrm{Hg}$. If restoration of central drive is important, the patient needs to spend the night, on average, with a respiratory alkalosis. This 
Table 1 Data from previously published trials (presented as non-invasive ventilation vs control group)

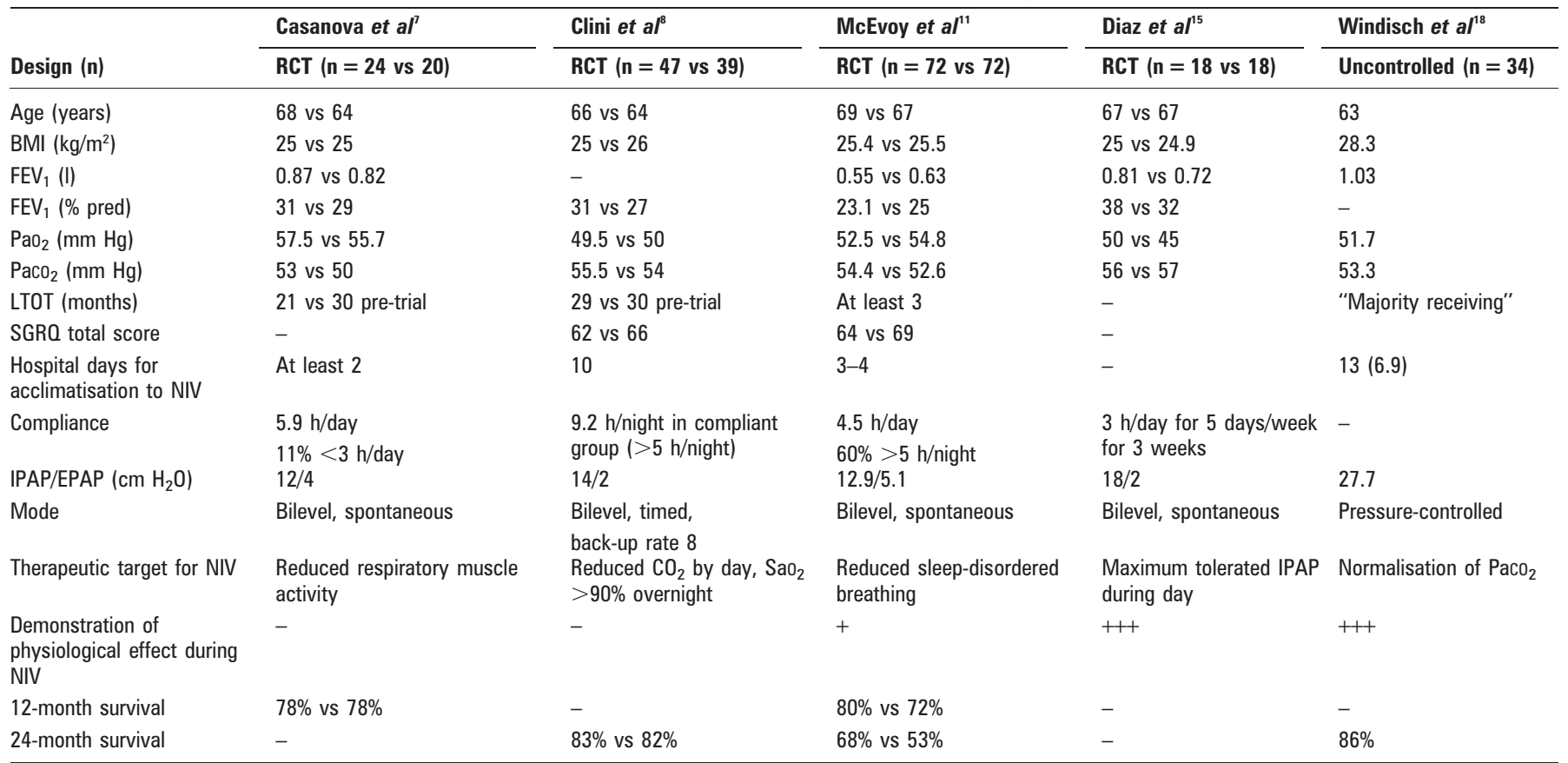

BMI, body mass index; EPAP, expiratory positive airway pressure; FEV $_{1}$, forced expiratory volume in $1 \mathrm{~s}$; IPAP, inspiratory positive airway pressure; LTOT, long-term oxygen therapy; NIV, noninvasive ventilation; $\mathrm{PaCO}_{2}, \mathrm{PaO}_{2}$, arterial carbon dioxide and oxygen tensions; RCT, randomised controlled trial; SGRQ, St George Respiratory Questionnaire.

will allow the excretion of bicarbonate and restoration of central drive. This requires the carbon dioxide tension to fall with NIV; if only the highest rises in carbon dioxide tension are reduced, this aim will not be achieved. The results of the bicarbonate concentration during daytime arterial blood gas measurements are not reported in this study, but would be expected to fall if carbon dioxide was reduced during NIV overnight.

Diaz and co-workers ${ }^{15}$ performed short-term (3 weeks) RCTs of daytime NIV compared with a control group who received sham ventilation. They gradually increased IPAP to the maximum that could be tolerated during the day and achieved an average of $18 \mathrm{~cm} \mathrm{H}_{2} \mathrm{O}$. This resulted in an approximate doubling of the tidal volume and halving of the respiratory rate. This would be expected to produce a substantial increase in alveolar ventilation and improve arterial blood gas tensions, although these data were not presented. They also showed a $48 \%$ reduction in the pressure time product of the diaphragm, indicating substantial unloading of the diaphragm. At the end of 3 weeks of ventilation there were improvements in both the $\mathrm{PaO}_{2}$ $\left(+8.6 \mathrm{~mm} \mathrm{Hg}\right.$ ) and the $\mathrm{PaCO}_{2}$ $(-8.4 \mathrm{~mm} \mathrm{Hg})$ during spontaneous breathing. There was a $93 \mathrm{ml}$ (13\%) increase in forced expiratory volume in $1 \mathrm{~s}\left(\mathrm{FEV}_{1}\right)$ and a reduction in hyperinflation, gas trapping and intrinsic positive end expiratory pressure (PEEP). Tidal volume during spontaneous breathing increased by $181 \mathrm{ml}$. In a separate study with a similar design ${ }^{17}$ they showed an increase in the 6-minute walking distance (mean $76 \mathrm{~m}$ ) and a reduction in dyspnoea scores. There were similar changes in arterial blood gas tensions during spontaneous breathing and an $80 \mathrm{ml}$ increase in $\mathrm{FEV}_{1}$ which correlated with the improved walking distance.

The study by Windisch et al, ${ }^{18}$ while uncontrolled, also provides some important insights into how NIV might be best used in patients with COPD. NIV was initiated in hospital and the level of inspiratory pressure support was gradually increased aiming for normocapnia, based on previous work showing that high inflation pressures could be tolerated resulting in improved $\mathrm{PaCO}_{2}$ during spontaneous breathing ${ }^{19}$ and also in QOL. ${ }^{20}$ Using this strategy, they showed that $\mathrm{PaCO}_{2}$ fell during spontaneous breathing by a mean of $7 \mathrm{~mm} \mathrm{Hg}, \mathrm{PaO}_{2}$ increased by a mean of $6 \mathrm{~mm} \mathrm{Hg}$ and $\mathrm{FEV}_{1}$ improved by a mean of $140 \mathrm{ml}$. The 2 -year survival rate was $86 \%$.

Taken together, these studies suggest that benefit is seen in daytime parameters (physiological and QOL) when there is a reduction in carbon dioxide during NIV. Given that NIV is usually applied during sleep, overnight monitoring is mandatory. End-tidal carbon dioxide measurements are of no use in patients with obstructive lung disease and monitoring of transcutaneous or arterial carbon dioxide tensions is required. Most of these studies used bilevel ventilators in spontaneous mode. Ventilators without a timed back-up are significantly cheaper and, in the absence of any data confirming that a timed backup is needed, these should be the machine of choice for domiciliary NIV in COPD. Higher inflation pressures appear to be necessary to affect physiological parameters.

The observation of improvements in $\mathrm{FEV}_{1}$ - often larger than those obtained with bronchodilators-is intriguing. For instance, in the UPLIFT Trial ${ }^{21}$ the mean improvement in $\mathrm{FEV}_{1}$ with tiotropium was $87-103 \mathrm{ml}$ over 4 years. In the TORCH Study ${ }^{22}$ the mean increase in $\mathrm{FEV}_{1}$ over 3 years with the combination of salmeterol and fluticasone was $92 \mathrm{ml}$. Diaz et $a l^{15}$ and Windisch et al ${ }^{19}$ both reported comparable increases in $\mathrm{FEV}_{1}$ after NIV. This raises the possibility that NIV has an effect on the airways themselves; possible mechanisms include a reduction in airway oedema ${ }^{23}$ or even stretching open of chronically fibrosed airways. If correct, it is likely that this will require a level of pressure to be delivered to the airways for a period of time. If this is an important mechanism, logic would suggest that the higher the pressure and the longer it is applied to the airways, the better. This is entirely speculative, but the improvements in $\mathrm{FEV}_{1}$ were only seen in these studies, ${ }^{15} 23$ both of which used higher inflation pressures. 
Although McEvoy et al showed an improvement in survival, this was apparently at the cost of worsening QOL with worse scores in generic questionnaires at 12 months. ${ }^{11}$ However, there are a number of difficulties with this. As pointed out by the authors, more patients in the non-invasive group completed OOL questionnaires than in the control group. It is also notable that only $50-60 \%$ of patients repeated the QOL questionnaire at 12 months. These factors may introduce "survivor" effects, which may introduce significant bias if patients with poor $\mathrm{QOL}$ do not feel well enough or choose not to fill in questionnaires. Second, many different domains of QOL were measured and no adjustment was made for multiple measures; there is a possibility that-by chance alone-one or more might be statistically significant. Finally, there is question about the choice of questionnaire. The disease-specific questionnaire (SGRQ) did not show a deterioration in QOL. Although the SGRO was developed in patients with milder disease, changes in QOL have been demonstrated in patients with COPD receiving NIV. ${ }^{6}$ The generic questionnaires did show a deterioration, and the SF36 has been shown to be reliable and responsive in COPD. ${ }^{24} 25$ Which measures should be believed? In other studies, ${ }^{6}{ }^{26}$ improvements in QOL have been shown to correlate with a reduction in bicarbonate, suggesting that effective control of nocturnal hypoventilation is important in improving quality of life. McEvoy et al showed an attenuation in the sleep-related rise in carbon dioxide, but the failure to lower carbon dioxide may explain the absence of a beneficial effect on QOL.

One other potential role for domiciliary NIV in patients with COPD is after discharge for an episode in which NIV has been used acutely in hospital. ${ }^{27}$ These patients have a poor prognosis, ${ }^{28}$ and there are some uncontrolled data to suggest that NIV may reduce the need for hospitalisation subsequently and be economically advantageous. ${ }^{29}$ In the study by McEvoy et al, ${ }^{11}$ hospitalisation rates did not differ between the two groups. However, it is difficult to interpret these data as the NIV + LTOT group had 7474 more days on the trial with a median follow-up of 28.5 months compared with 20.5 months in the LTOT alone group. It may not be appropriate to compare raw rates in a progressive disease such as COPD; patients are probably more likely to be admitted when the condition is more advanced. In the study by Clini et $a l^{8}$ hospital admissions did not differ between the groups during follow-up. However, in a post hoc analysis comparing the year before with the first year of the trial, hospital admissions decreased by $45 \%$ in the NIV + oxygen group and increased by $27 \%$ in the oxygen group. This issue warrants further investigation in future trials.

What can be concluded about the current role for NIV in patients with stable COPD following the study of McEvoy et al? First, that ventilation for an average of $4 \mathrm{~h} /$ night with modest inflation pressures applied with the intent of reducing sleep-disordered breathing reduced the highest peaks of $\mathrm{TcCO}_{2}$ and improved survival. The benefit was lost by 3.5 years. Second, this improvement in survival may have been at the expense of QOL. The results from different studies using different questionnaires are conflicting and future studies should use questionnaires validated in patients with COPD with chronic respiratory failure receiving mechanical ventilation such as the SRI or MRF28 ${ }^{20}$; if multiple questionnaires are used there must be a clearly defined analysis plan from the outset. Third, future trials must have a clear therapeutic end point during NIV and it must be confirmed objectively that this is achieved. Available data suggest that this should be a demonstration of a reduction in the carbon dioxide level overnight. This should be to a level that allows net excretion of bicarbonate and restoration of central drive. It is likely that higher pressures will be needed to achieve this. The possibility that NIV has a direct effect on the airways needs to be explored in future studies and higher pressures may be important in this regard. The case for domiciliary NIV in patients with COPD remains unproven, and most patients with COPD should probably only be offered NIV in the context of a clinical trial. However, there is sufficient evidence of benefit to suggest that trials of domiciliary NIV for COPD should be funded, as COPD continues to be a major cause of morbidity and incurs high costs for healthcare providers. In the absence of such trials, it is reasonable for hypercapnic patients with COPD, already established on LTOT, to be offered domiciliary NIV.

\section{Competing interests: None.}

Thorax 2009;64:553-556. doi:10.1136/thx.2009.113423

\section{REFERENCES}

1. Lloyd-Owen SJ, Donaldson GC, Ambrosino N, et al. Patterns of home mechanical ventilation use in Europe: results from the Eurovent survey. Eur Respir $J$ 2005;25:1025-31.
2. Gay PC, Hubmayr RD, Stroetz RW. Efficacy of nocturnal nasal ventilation in stable, severe chronic obstructive pulmonary disease during a 3-month controlled trial. Mayo Clin Proc 1996;71:533-42.

3. Strumpf DA, Millman RP, Carlisle CC, et al. Nocturnal positive-pressure ventilation via nasal mask in patients with severe chronic obstructive pulmonary disease. Am Rev Respir Dis 1991;144:1234-9.

4. Lin CC. Comparison between nocturnal nasal positive pressure ventilation combined with oxygen therapy and oxygen monotherapy in patients with severe COPD. Am J Respir Crit Care Med 1996;154:353-8.

5. Wijkstra PJ, Lacasse $Y$, Guyatt $\mathrm{GH}$, et al. A metaanalysis of nocturnal noninvasive positive pressure ventilation in patients with stable COPD. Chest 2003; 124:337-43.

6. Meecham Jones DJ, Paul EA, Jones PW, et al. Nasal pressure support ventilation plus oxygen compared with oxygen therapy alone in hypercapnic COPD. Am J Respir Crit Care Med 1995;152:538-44.

7. Casanova C, Celli BR, Tost L, et al. Long-term controlled trial of nocturnal nasal positive pressure ventilation in patients with severe COPD. Chest 2000;118:1582-90.

8. Clini E, Sturani C, Rossi A, et al. The Italian multicentre study on noninvasive ventilation in chronic obstructive pulmonary disease patients. Eur Respir J 2002;20:529-38.

9. Garrod R, Mikelsons C, Paul EA, et al. Randomized controlled trial of domiciliary noninvasive positive pressure ventilation and physical training in severe chronic obstructive pulmonary disease. Am J Respir Crit Care Med 2000;162:1335-41.

10. Duiverman ML, Wempe JB, Bladder G, et al. Nocturnal non-invasive ventilation in addition to rehabilitation in hypercapnic patients with COPD. Thorax 2008;63:1052-7.

11. McEvoy RD, Pierce RJ, Hillman D, et al. Nocturnal non-invasive nasal ventilation in stable hypercapnic COPD : a randomised controlled trial. Thorax 2009;64:561-6.

12. Nickol AH, Hart N, Hopkinson NS, et al. Mechanisms of improvement of respiratory failure in patients with restrictive thoracic disease treated with non-invasive ventilation. Thorax 2005;60:754-60.

13. Annane D, Quera-Salva MA, Lofaso F, et al. Mechanisms underlying effects of nocturnal ventilation on daytime blood gases in neuromuscular diseases. Eur Respir J 1999;13:157-62.

14. Turkington PM, Elliott MW. Rationale for the use of non-invasive ventilation in chronic ventilatory failure. Thorax 2000;55:417-23.

15. Diaz 0, Begin P, Torrealba B, et al. Effects of noninvasive ventilation on lung hyperinflation in stable hypercapnic COPD. Eur Respir J 2002;20:1490-8.

16. Fu ES, Downs JB, Schweiger JW, et al. Supplemental oxygen impairs detection of hypoventilation by pulse oximetry. Chest 2004; 126:1552-8.

17. Diaz 0, Begin $\mathrm{P}$, Andresen $\mathrm{M}$, et al. Physiological and clinical effects of diurnal noninvasive ventilation in hypercapnic COPD. Eur Respir J 2005;26:1016-23.

18. Windisch W, Kostic S, Dreher M, et al. Outcome of patients with stable COPD receiving controlled noninvasive positive pressure ventilation aimed at a maximal reduction of $\mathrm{Pa}(\mathrm{CO} 2)$. Chest 2005;128:657-62.

19. Windisch W, Vogel M, Sorichter S, et al. Normocapnia during nIPPV in chronic hypercapnic COPD reduces subsequent spontaneous $\mathrm{PaCO}_{2}$ Respir Med 2002;96:572-9.

20. Windisch W, Budweiser S, Heinemann F, et al. The Severe Respiratory Insufficiency Questionnaire was valid for COPD patients with severe chronic respiratory failure. J Clin Epidemiol 2008;61:848-53.

21. Tashkin DP, Celli B, Senn S, et al. A 4-year trial of tiotropium in chronic obstructive pulmonary disease. N Engl J Med 2008;359:1543-54.

22. Calverley PM, Anderson JA, Celli B, et al. Salmeterol and fluticasone propionate and survival in chronic obstructive pulmonary disease. N Engl J Med 2007;356:775-89. 
23. Burns GP, Gibson GJ. A novel hypothesis to explain the bronchconstrictor effect of deep inspiration in asthma. Thorax 2002; 57:116-9.

24. Mahler DA, Mackowiak Jl. Evaluation of the shortform 36-item questionnaire to measure health-related quality of life in patients with COPD. Chest 1995:107:1585-9.

25. Moy ML, Ingenito EP, Mentzer SJ, et al. Healthrelated quality of life improves following pulmonary rehabilitation and lung volume reduction surgery Chest 1999;115:383-9.

26. Windisch W, Dreher M, Storre JH, et al. Nocturnal non-invasive positive pressure ventilation: physiological effects on spontaneous breathing. Respir Physiol Neurobiol 2006;150:251-60.

27. Elliott MW. Non-invasive ventilation in acute exacerbations of COPD: what happens after hospital discharge? Thorax 2004;59:1006-8.
28. Chu CM, Chan VL, Lin AWN, et al. Readmission rates and life threatening events in COPD survivors treated with non-invasive ventilation for acute hypercapnic respiratory failure. Thorax 2004;59:1020-5.

29. Tuggey JM, Plant PK, Elliott MW. Domiciliary noninvasive ventilation for recurrent acidotic exacerbations of COPD: an economic analysis. Thorax 2003;58:867-71.

\section{Biomarkers and community- acquired pneumonia}

\section{Jeremy S Brown}

Community-acquired pneumonia (CAP) is common, and the delivery of optimum care to patients with CAP is important to limit the associated substantial mortality and morbidity. Although severity assessment of patients presenting with CAP using clinical scores such as the CURB65 (Confusion, Urea nitrogen, Respiratory rate, Blood pressure, 65 years of age and older) or Pneumonia Severity Index (PSI) scales aids management, there are many other areas in which the care of patients with CAP could be improved. For example, improved accuracy of identifying patients at risk of death (especially the significant proportion of deaths in patients with low clinical severity scores) or complications such as empyema would improve the stratification of patients for different intensities of management, and there are few data on how to decide the duration of antibiotic treatment for individual patients. Biomarkers may help with some of these questions, and in this month's Thorax there are two articles (see pages 587 and pages 592 on the use of biomarkers in patients with $\mathrm{CAP}^{12}$ that join a series of often seemingly contradictory papers in this area published over the past few years. What are the main overall messages that can be concluded from the published research into biomarkers and CAP, and are their results convincing enough now to influence how patients with CAP are managed?

The National Institutes of Health definition of a biomarker is: "a characteristic that is objectively measured and evaluated as an indicator of normal biologic processes, pathogenic processes,

Correspondence to: Dr Jeremy S Brown, Centre for Respiratory Research, Department of Medicine, University College Medical School, Rayne Institute, London WC1E 6JJ, UK; jeremy.brown@ucl.ac.uk or pharmacologic responses to a therapeutic intervention." However, this definition would include clinical parameters such as temperature or respiratory rate, and in general a biomarker is given a narrower definition of a biochemical feature that can be used to diagnose or assess the progress of disease or the effects of treatment. As such, blood urea levels for the CURB65 score and $\mathrm{PaO}_{2}$ (arterial oxygen tension), $\mathrm{pH}$ and blood chemistry results for the PSI are examples of biomarkers already incorporated into guidelines for the management of CAP. In contrast, most recent research into biomarkers for CAP has concentrated on the markers of inflammation C-reactive protein (CRP) and procalcitonin (PCT). ${ }^{1-8}$ Both CRP and PCT are acute phase reactants with low circulating levels normally, but which rapidly increase during inflammatory disease sometimes to markedly high levels. CRP is produced by the liver in response to proinflammatory cytokines (especially interleukin 6 (IL6)), and PCT is produced by the liver, kidneys and monocytes after stimulation by proinflammatory cytokines and by bacterial products. Importantly, both can be measured relatively cheaply and quickly, although CRP measurement is routinely available more widely than PCT. Various cytokines (eg, tumour necrosis factor $\alpha$, IL1, IL6, IL8 and IL10), free cortisol and a range of alternative prohormones to PCT (eg, proatrial natiuretic peptide, provasopressin and proadrenomedullin) have also been assessed as biomarkers of inflammation in patients with CAP, ${ }^{2-11}$ but the data on these are limited compared with those for CRP and PCT, and their measurement is generally not routinely available.

What are the potential roles for biomarkers of inflammation in managing CAP? Biomarkers could be helpful in several areas, including: (1) confirming the diagnosis of CAP; (2) identifying the potential causative agent; (3) assessing severity, mortality risk and the potential for complications; and (4) identifying when a patient is better, or conversely identifying those with complications. In addition, biomarkers may be able to identify which severely unwell patients are most likely to benefit from new therapeutic interventions based on immunomodulation, such as activated protein C treatment, but at present this is not possible and will be discussed no further in this editorial.

A significant infection such as CAP should almost always lead to the release of proinflammatory cytokines, which will in turn cause an increase in biomarkers of inflammation. Biomarkers of inflammation would perhaps then be a more reliable indicator of infection than a pyrexia or raised white cell count, and several studies have looked at the utility of CRP and PCT for assisting the diagnosis of CAP.8 ${ }^{12}$ These studies suggest that, as expected, a diagnosis of CAP is unlikely unless there has been a significant increase in CRP or PCT (eg, $>40 \mathrm{mg} / \mathrm{l}$ or $0.1 \mu \mathrm{g} / \mathrm{l}$, respectively). Conversely, in patients with a clinical suspicion of CAP the diagnosis is very likely if the patient has very high CRP or PCT levels (eg, >200 mg/l or $1.0 \mu \mathrm{g} / \mathrm{l}$, respectively). ${ }^{8}$ However, in most patients, a clinical diagnosis of CAP is not difficult and will often be made without the benefit of blood test results, so the utility of a biomarker of inflammation to support the diagnosis of CAP may be limited to specific situations. These might include patients who are apyrexial at presentation, in whom a raised CRP or PCT could confirm the possibility of infection, or patients with underlying parenchymal lung diseases such as idiopathic pulmonary fibrosis in whom the clinical or radiological evaluation of the presence of new consolidation is difficult. At present, data on the role of biomarkers in these specific clinical scenarios are lacking.

Biomarkers of inflammation may help indicate the causative organism in CAP. 